$\Rightarrow$ CHROMATIN

\section{A positive twist}

Centromeric nucleosomes are characterized by the presence of one or more centromeric histone H3 (CenH3; known as CID in Drosophila melanogaster and Cse4 in Saccharomyces cerevisiae) variants that replace canonical histone H3. However, little is known about the structural features of centromeres

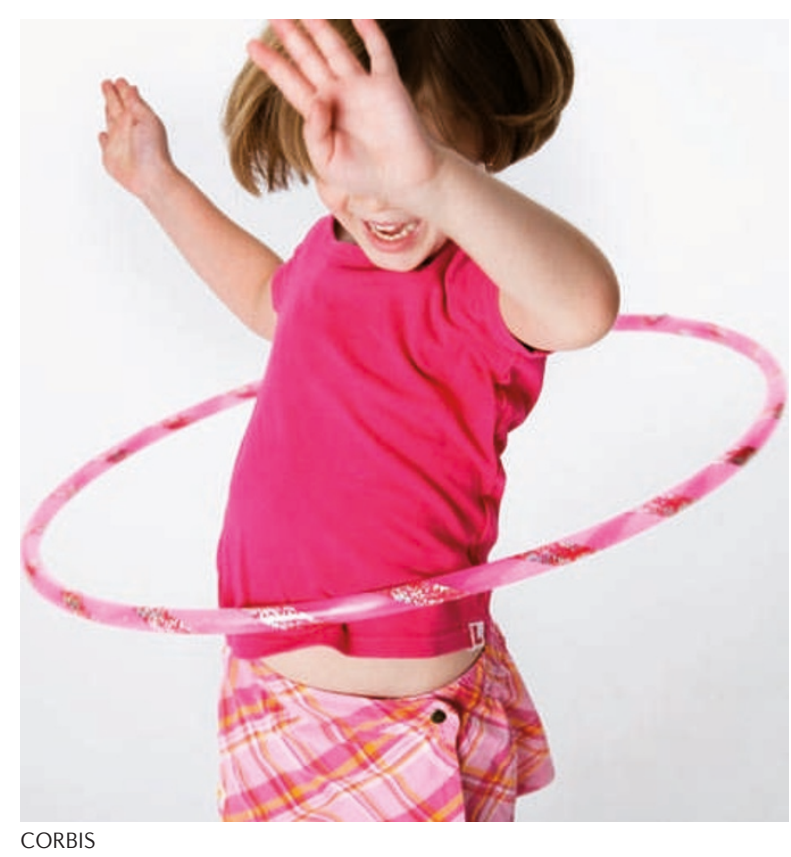

that enable them to recruit kinetochore components and be faithfully inherited. Furuyama and Henikoff now report the surprising finding that, whereas $\mathrm{H} 3$-containing nucleosomes wrap DNA in a lefthanded manner and induce negative supercoils, CenH3-containing nucleosomes from fruit fly and budding yeast induce positive supercoils, implying the right-handed wrapping of DNA around nucleosomes.

By analysing CenH3 nucleosomes reconstituted in vitro from $D$. melanogaster histones in a plasmid supercoiling assay, the authors showed that $\mathrm{CenH} 3$ nucleosomes induce positive supercoils. By inserting various DNAs in a plasmid vector, they concluded that the direction of supercoiling depends on the presence of CenH3-containing nucleosomes rather than the properties of specific DNA sequences.

Given that an in vivo centromere system in $D$. melanogaster would be impractical for various reasons, the authors developed an S. cerevisiae minichromosome system containing either a single functional yeast centromere (wild type) or no centromere (mutant). Minichromosomes isolated from wild-type and mutant strains were assayed: each $\mathrm{H} 3$-containing nucleosome induced a negative supercoil in both strains, whereas the centromere present in the wild-type strain induced a positive supercoil and 'cancelled' a negative supercoil by replacing an $\mathrm{H} 3$-containing nucleosome (so, the net difference between having a centromere and not having one is two supercoils). Using a kinetochore-defective yeast strain, the authors further showed that kinetochores do not influence the topological state of the minichromosomes.

Furuyama and Henikoff conclude that positive supercoiling, which indicates right-handed wrapping of DNA around the histone core, occurs in CenH3-containing nucleosomes. They further suggest that centromeric nucleosomes probably exist as tetrameric 'hemisomes', although the composition of the positively supercoiled chromatin remains to be established in vitro and in vivo. The authors propose that "positive supercoiling is a general feature of centromeric nucleosomes that has important implications for maintaining centromeres".

Arianne Heinrichs

ORIGINAL RESEARCH PAPER Furuyama, T. $\delta$ Henikoff, S. Centromeric nucleosomes induce positive DNA supercoils. Cell 138, 104-113 (2009) 